\title{
Lifting idempotents and Clifford theory
}

\author{
JACQUES THÉVENAZ
}

Let $N$ be a normal subgroup of a finite group $G$ and let $R$ be a noetherian complete local commutative ring. Clifford theory deals with the relationship between $R G$-modules and $R N$-modules, using induction from $N$ to $G$ or restriction from $G$ to $N$. Since Clifford's 1937 paper [1], the theory is well understood for irreducible representations (see also $[2, \S 11 \mathrm{C}]$ ). For an indecomposable $R N$ module $W$, several authors have proved a going-up theorem describing how $\operatorname{Ind}_{N}^{G} W$ decomposes (see $[2, \S 19 C]$ ).

One purpose of this paper is to prove (in Section 2) a going-down theorem for indecomposable modules (analogous to Clifford's theorem), based on a refinement of the lifting idempotents theorem, presented in Section 1. The going-up and going-down theorems are actually equivalent in the sense that each can be derived as a corollary to the other one. One main assumption is necessary for the going-down theorem: the $R G$-module we start from must be projective relative to $H$. The whole procedure is presented in the more general context of Clifford systems. The paper concludes in Section 3 with another application of the lifting idempotents theorem, concerning the behaviour of indecomposable modules under ground ring extensions.

\section{Lifting idempotents}

THEOREM 1. Let $A$ be $a$ ring and $J$ a two-sided ideal contained in $\operatorname{Rad} A$. Assume that $A$ is complete in the $J$-adic topology (that is the natural map $A \rightarrow$ $\lim A / J^{n}$ is an isomorphism). Let $\Pi$ be a finite group acting on $A$ by automorphisms leaving $J$ globally invariant. Let $\left\{\bar{e}_{1}, \ldots, \bar{e}_{n}\right\}$ be a set of orthogonal idempotents of $\bar{A}=A / J$ satisfying $\sum_{i=1}^{n} \bar{e}_{i}=1$. Assume the following three conditions:

a) The induced action of $I$ on $\bar{A}$ permutes the idempotents $\bar{e}_{i}$ transitively.

b) There exists $u \in A$ such that $\operatorname{Tr}_{\Omega}(u)=1$ where $\Omega$ is the stabilizer of $\bar{e}_{1}$ and $\operatorname{Tr}_{\boldsymbol{\Omega}}(u)=\sum_{\omega \in \Omega} \omega u$.

c) $\bar{u}$ commutes with each $\overline{\boldsymbol{e}}_{\mathrm{i}}$.

Then $\left\{\bar{e}_{1}, \ldots, \tilde{e}_{n}\right\}$ lifts to a set $\left\{e_{1}, \ldots, e_{n}\right\}$ of orthogonal idempotents of $A$ which are permuted by $\Pi$ transitively and such that $\sum_{i=1}^{n} e_{i}=1$. 
Remarks. 1) If $A$ is the ring of endomorphisms of a representation $V$, we shall see that the condition b) corresponds to a condition of relative projectivity for $V$.

2) There are two situations where c) is always satisfied: either the idempotents $\bar{e}_{i}$ are central or the order $|\Omega|$ of $\Omega$ is invertible in $A$ in which case one can choose $u$ to be the central element $|\Omega|^{-1}$.

3) When $\Pi$ acts regularly on the idempotents $\bar{e}_{i}$, that is when $\Omega$ is trivial, one can take $u=1$ so that $b$ ) and c) are trivially satisfied. This special case appears already in [3].

Proof. It suffices to prove the theorem when $J$ is nilpotent because, since $A \cong \lim A / J^{n}$, the lifted idempotents are constructed as limits of idempotents of $A / J^{n}$ for $n \rightarrow \infty$.

For $\sigma \in \Pi$, write $\bar{e}_{\sigma}=\sigma \bar{e}_{1}$ so that $\bar{e}_{\sigma}=\bar{e}_{\tau}$ if and only if $\sigma \Omega=\tau \Omega$. Since $\Pi$ acts transitively, every idempotent $\bar{e}_{i}$ can be written in that form.

We proceed by induction on the nilpotent index $n$ of $J$. There is nothing to prove if $n=1$. If $n \geqslant 2$, let $I=J^{n-1}$ and write $\tilde{a}$ for the image of $a \in A$ modulo $I$. By induction, there exist idempotents $\tilde{e}_{\sigma}$ of $A / I$ such that $\sigma \tilde{e}_{\tau}=\tilde{e}_{\sigma \tau}$ and $\sum_{\sigma \in \Pi / \Omega} \tilde{e}_{\sigma}=1$. First lift arbitrarily the idempotents $\tilde{e}_{\sigma}$ to get orthogonal idempotents $e_{\sigma}$ of $A$ satisfying $\sum_{\sigma \in \Pi / \Omega} e_{\sigma}=1$. This is well known to be possible (see $[2, \S 6 \mathrm{~A}])$. Of course the notation implies that we keep the convention:

$$
e_{\sigma}=e_{\tau} \quad \text { if and only if } \sigma \Omega=\tau \Omega .
$$

Since $\boldsymbol{\sigma} \tilde{\boldsymbol{e}}_{\tau}=\tilde{\boldsymbol{e}}_{\boldsymbol{\sigma r}}$, we have:

$$
\sigma e_{\tau}=e_{\sigma \tau}+r_{\sigma, \tau} \text { for some } r_{\sigma, \tau} \in I \text {. }
$$

We list several properties of the elements $r_{\sigma, \tau}$ :

(1) If $\omega \in \Omega, r_{\sigma, \tau \omega}=r_{\sigma, \tau}$.

This follows from $e_{n \omega}=e_{\eta}$ for all $\eta \in \Pi$.

(2) $\sum_{\tau \in \Pi / \Omega} r_{\sigma, \tau}=0$.

This follows when $\sigma$ is applied to $1=\sum_{\tau \in \Pi / \Omega} e_{\tau}$.

(3) $\eta r_{\sigma, \tau}=r_{n \sigma, \tau}-r_{\eta, \sigma r}$

This is a consequence of $(\eta \sigma) e_{\tau}=\eta\left(\sigma e_{\tau}\right)$.

(4) $r_{\sigma, \tau}=e_{\sigma \tau} r_{\sigma, \tau}+r_{\sigma, \tau} e_{\sigma \tau}$ 
This follows from the equality $\sigma e_{\tau}=\left(\sigma e_{\tau}\right)^{2}$ using also $I^{2}=0$. Multiplying (4) by $e_{\eta}$ on the right or $e_{\lambda}$ on the left (or both in the first case below), we get:

(5) $e_{\lambda} r_{\sigma, \tau} e_{\eta}=\left\{\begin{array}{lll}0 & \text { if } & \lambda \Omega \neq \sigma \tau \Omega \neq \eta \Omega \\ r_{\sigma, \tau} e_{\eta} & \text { if } & \lambda \Omega=\sigma \tau \Omega \neq \eta \Omega \\ e_{\lambda} r_{\sigma, \tau} & \text { if } & \lambda \Omega \neq \sigma \tau \Omega=\eta \Omega \\ 0 & \text { if } & \lambda \Omega=\sigma \tau \Omega=\eta \Omega\end{array}\right.$

(6) If $\lambda \Omega \neq \eta \Omega, e_{\mu \lambda} r_{\mu, \eta}+r_{\mu, \lambda} e_{\mu \eta}=0$.

This is a consequence of $\left(\mu e_{\lambda}\right) \cdot\left(\mu e_{\eta}\right)=0$ using again $I^{2}=0$.

Now define: $f_{\sigma}=e_{\sigma}+\sum_{\lambda \in I} r_{\lambda, \lambda^{-1} \sigma} \cdot e_{\lambda} \cdot \lambda u$ where $u \in A$ satisfies hypotheses b) and c). By (1), we have:

(7) $f_{\sigma \omega}=f_{\sigma}$ if $\omega \in \Omega$.

(8) $\sum_{\sigma \in \Pi / \Omega} f_{\sigma}=1$.

For

$$
\begin{aligned}
\sum_{\sigma \in \Pi / \Omega} f_{\sigma} & =\sum_{\sigma \in \Pi / \Omega} e_{\sigma}+\sum_{\sigma \in \Pi / \Omega} \sum_{\lambda \in \Pi} r_{\lambda, \lambda^{-1} \sigma} \cdot e_{\lambda} \cdot \lambda u \\
& =1+\sum_{\lambda \in \Pi}\left(\sum_{\sigma \in \Pi / \Omega} r_{\lambda, \lambda^{-1} \sigma}\right) e_{\lambda} \cdot \lambda u=1 \quad \text { by (2). }
\end{aligned}
$$

(9) $f_{\sigma} f_{\tau}=0$ if $\sigma \Omega \neq \tau \Omega$.

$$
f_{\sigma} f_{\tau}=\sum_{\lambda \in \Pi} e_{\sigma} r_{\lambda, \lambda{ }^{-1} \tau} e_{\lambda} \cdot \lambda u+\sum_{\lambda \in \Pi} r_{\lambda, \lambda^{-1} \sigma} e_{\lambda} \cdot \lambda u \cdot e_{\tau}
$$

By hypothesis c), $\lambda \bar{u} \cdot \bar{e}_{\tau}=\lambda\left(\bar{u} \cdot \bar{e}_{\lambda^{-1} \tau}\right)=\lambda\left(\bar{e}_{\lambda^{-1} \tau} \cdot \bar{u}\right)=\bar{e}_{\tau} \cdot \lambda \bar{u}$. Hence $\lambda u$ commutes with $e_{\tau}$ modulo $J$. Since $I \cdot J=J^{n-1} \cdot J=0$, we have $r \cdot \lambda u \cdot e_{\tau}=r \cdot e_{\tau} \cdot \lambda u$ for all $r \in I$ and so we can permute $\lambda u$ and $e_{\tau}$ in the second sum. Therefore, the only non-zero terms appear for $\lambda \in \tau \Omega$. By (5), the same holds for the first sum. Consequently:

$$
f_{\sigma} f_{\tau}=\sum_{\omega \in \Omega}\left(e_{\sigma} r_{\tau \omega, \omega^{-1}}+r_{\tau \omega, \omega^{-1} \tau^{-1} \sigma} e_{\tau \omega}\right) e_{\tau \omega} \cdot \tau \omega u
$$

Now apply (6) with $\eta=1, \mu=\tau \omega$ and $\lambda=\omega^{-1} \tau^{-1} \sigma$, using also (1). The condition $\lambda \Omega \neq \eta \Omega$ is equivalent to $\sigma \Omega \neq \tau \Omega$. We get $f_{\sigma} f_{\tau}=0$, as required.

Clearly (8) and (9) imply that $f_{\sigma}$ is idempotent. There remains to prove the additional property we are looking for:

(10) $\tau f_{\sigma}=f_{\tau \sigma}$ 
By (3), we have:

$$
\tau f_{\sigma}=e_{\tau \sigma}+r_{\tau, \sigma}+\sum_{\lambda \in \Pi}\left(r_{\tau \lambda, \lambda}^{-1} \sigma-r_{\tau, \sigma}\right) \cdot\left(e_{\tau \lambda}+r_{\tau, \lambda}\right) \cdot \tau \lambda u .
$$

Since $I^{2}=0$, we get:

$$
\begin{aligned}
\tau f_{\sigma} & =e_{\tau \sigma}+\sum_{\lambda \in \Pi} r_{\tau \lambda, \lambda^{-1} \sigma} \cdot e_{\tau \lambda} \cdot \tau \lambda u+r_{\tau, \sigma}\left(1-\sum_{\lambda \in \Pi \Pi} e_{\tau \lambda} \cdot \tau \lambda u\right) \\
& =e_{\tau \sigma}+\sum_{\mu \in \Pi} r_{\mu, \mu^{-1} \tau \sigma} \cdot e_{\mu} \cdot \mu u+r_{\tau, \sigma}\left(1-\sum_{\mu \in I I / \Omega} e_{\mu} \cdot \sum_{\omega \in \Omega} \mu \omega u\right) \\
& =f_{\tau \sigma}+r_{\tau, \sigma}\left(1-\sum_{\mu \in \Pi \Pi / \Omega} e_{\mu} \cdot \mu T r_{\Omega}(u)\right)=f_{\tau \tau r}
\end{aligned}
$$

using $\operatorname{Tr}_{\Omega}(u)=1$ and $\sum_{\mu \in I I / \Omega} e_{\mu}=1$.

\section{Clifford theory}

Let $N$ be a normal subgroup of a finite group $G$ and $S=G / N$. Throughout this section, $R$ denotes a noetherian local commutative ring which is complete in its natural topology of local ring. These assumptions are made in order to have the following properties:

(i) Every finitely generated $R G$-module is a direct sum of indecomposable submodules.

(ii) If $M$ is an indecomposable $R G$-module, then End $_{R G} M$ is a local ring. Hence Krull-Schmidt theorem holds for RG-modules.

In order to study the restriction to $N$ of an indecomposable $R G$-module, we consider the more general case of an $S$-graded Clifford system $A=\bigoplus_{\mathrm{sES}} A_{\mathrm{s}}$ over $R$, in the sense of $[2, \S 11 \mathrm{C}]$. The case of group algebras corresponds to $A=R G$ and $A_{1}=R N$. Recall that there exist units $a_{s} \in A_{s}$ such that $A_{s}=a_{s} A_{1}=A_{1} a_{s}$. Also $a_{s} a_{t} a_{s t}^{-1} \in A_{1}$ because $A_{s} A_{t}=A_{s t}$.

For the rest of this paper, all modules will be finitely generated left modules. For an $A_{1}$-module $W$, denote by $W^{A}$ the induced module Ind $_{A_{1}}^{A} W=A \otimes_{A_{1}} W$, while for an $A$-module $V$, we denote by $V_{A_{1}}$ the restriction $\operatorname{Res}_{A_{1}}^{A_{1}} V$. If $V$ is an $A$-module, then $S$ acts on End $\mathrm{A}_{A_{1}} V$ by $s f=a_{s} f a_{s}^{-1}$ and the set of fixed points is exactly $\operatorname{End}_{\mathrm{A}} V$.

DEFINITIONS. 1) An A-module $V$ is said to be projective relative to $A_{1}$ if $V$ is a direct summand of a module induced from $A_{1}$ which actually can be chosen to 
be $\left(V_{A_{1}}\right)^{A}$. This is equivalent to the existence of an endomorphism $u \in \operatorname{End}_{A_{1}} V$ such that $\operatorname{Tr}_{S}(u)=1$ where $\operatorname{Tr}_{S}(u)=\sum_{s \in S} s u$. The equivalence of these definitions is well known in the case of group algebras [2, $\$ 19 \mathrm{~A}]$, but the proof can be carried over without change to the case of Clifford systems.

2) If $W$ is an $A_{1}$-module, then $a_{s} \otimes W$ has a natural structure of $A_{1}$-module and is called a conjugate of $W$.

3) Let $M=\oplus_{i, j} M_{i j}$ be a decomposition of a module $M$ into indecomposable summands such that $M_{i j} \cong M_{i k}$ for all $i, j, k$ and $M_{i j} \not \equiv M_{k m}$ if $i \neq k$. Then $M_{i}=$ $\bigoplus_{j} M_{i j}$ is called a homogeneous component of $M$. Contrary to the case of semisimple modules, note that in general $M_{i}$ is not uniquely determined by $M$.

Now we can state the going-down theorem analogous to Clifford's theorem:

THEOREM 2. Let $A$ be an $S$-graded Clifford system over $R$ and $V$ an indecomposable $A$-module. Assume that $V$ is projective relative to $A_{1}$, that is there exists an indecomposable summand $W$ of $V_{A_{1}}$ such that $V$ is a direct summand of $W^{A}$. Let $T=\left\{t \in S \mid a_{t} \otimes W \cong W\right\}$ be the inertial subgroup of $W$ and let $\left\{s_{1}, \ldots, s_{n}\right\}$ be a set of coset representatives of $T$ in $S$. Finally let $B=\bigoplus_{t \in T} A_{t}$ be the $T$-graded subalgebra of $A$. Then:

(i) $V_{A_{1}}$ is isomorphic to a direct sum of conjugates of $W$.

(ii) $\left\{a_{s_{i}} \otimes W \mid i=1, \ldots, n\right\}$ is a complete set of non-isomorphic conjugates of $W$ and each appears with the same multiplicity in a decomposition of $V_{\mathrm{A}_{1}}$.

(iii) There exists a decomposition $V_{A_{1}}=\bigoplus_{i=1}^{n} U_{i}$ into homogeneous components which are permuted transitively by $\left\{a_{s} \mid s \in S\right\}$ and such that $\left\{a_{t} \mid t \in T\right\}$ stabilizes $U_{1}$.

(iv) $U_{1}$ is an indecomposable $B$-module and $V$ is isomorphic to $U_{1}^{\mathrm{A}}$.

Beside Theorem 1, the main ingredient for the proof of Theorem 2 is the following:

PROPOSITION 3. Let $\boldsymbol{A}$ be an $\boldsymbol{R}$-algebra, finitely generated as $\boldsymbol{R}$-module, and $M$ an $A$-module. Denote by a bar the reduction modulo the radical of $\operatorname{End}_{A} M$. Let $M=\bigoplus_{i=1}^{n} M_{i}$ (respectively $M=\bigoplus_{i=1}^{n} M_{i}^{\prime}$ ) be any decomposition of $M$ corresponding to idempotents $e_{1}, \ldots, e_{n} \in$ End $_{\mathrm{A}} M$ (respectively $e_{1}^{\prime}, \ldots, e_{n}^{\prime}$ ).

(i) The modules $M_{i}$ are homogeneous components of $M$ if and only if $\bar{e}_{1}, \ldots, \bar{e}_{n}$ are the primitive central idempotents of $\overline{\operatorname{End}_{\mathrm{A}} M}$.

(ii) Assume the modules $M_{i}$ and $M_{i}^{\prime}$ are homogeneous components of $M$, labelled in order to have $M_{i} \cong M_{i}^{\prime}$ for all $i$. Then there exists $f \in \operatorname{Aut}_{A} M$ such that $f\left(M_{i}\right)=M_{i}^{\prime}$ for all $i$ and $\bar{f}=1$.

(iii) Assume the modules $M_{i}$ and $M_{i}^{\prime}$ are homogeneous components of $M$. Then $M_{1} \cong M_{1}^{\prime}$ if and only if $\bar{e}_{1}=\bar{e}_{1}^{\prime}$. 
Proof. (i) If the modules $M_{i}$ are homogeneous components of $M$, write $M_{i} \cong m_{i} N_{i}$ with $N_{i}$ indecomposable. Let $E_{i}=\operatorname{End}_{\mathrm{A}} N_{i}$ and $D_{i}=\overline{\operatorname{End}_{\mathrm{A}} N_{i}}$. By Fitting's theorem $[2, \S 19 \mathrm{C}$, lemma], there is a commutative diagram

$$
\prod_{i=1}^{n} M_{m_{1}}\left(E_{i}\right) \cong \prod_{i=1}^{n} \operatorname{End}_{\mathrm{A}} M_{i} \operatorname{End}_{\mathrm{A}} M
$$

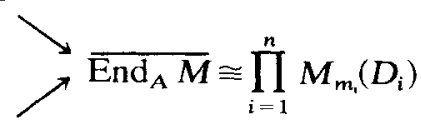

Since $e_{i}$ is the unit matrix of $M_{m_{1}}\left(E_{i}\right)$ (with zeros in all other components), $\bar{e}_{i}$ is the unit matrix of $M_{m_{i}}\left(D_{i}\right)$, i.e. $\bar{e}_{i}$ is a primitive central idempotent of $\overline{\text { End }_{\mathrm{A}} M}$.

If conversely $\bar{e}_{i}$ is primitive central, decompose it into primitive idempotents $\bar{e}_{i}=\bar{e}_{i 1}+\cdots+\bar{e}_{i m_{1}}$ and lift them to get $e_{i}=e_{i 1}+\cdots+e_{i m_{1}}$. Now $e_{i j} E \cong e_{i k} E$ because $\bar{e}_{i j} \bar{E} \cong \bar{e}_{i k} \bar{E}$. Therefore:

$$
e_{i j} M \cong e_{i j} E \otimes_{E} M \cong e_{i k} E \otimes_{E} M \cong e_{i k} M .
$$

So $e_{i} M=\bigoplus_{i=1}^{m_{i}} e_{i j} M$ is a homogeneous decomposition of $e_{i} M$ into indecomposable summands. If some indecomposable summand of $e_{i} M$ was isomorphic to a summand of $e_{k} M$ for $k \neq i$, there would be less than $n$ homogeneous components in $M$ and so, by the first part of the proof, less than $n$ primitive central idempotents in $\bar{E}$.

(ii) Consider again the commutative diagram

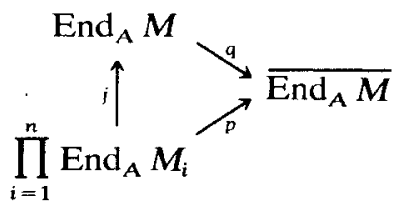

We emphasize that not only $q$ but also $p$ is surjective. Choose an isomorphism $g_{i}: M_{i} \rightarrow M_{i}^{\prime}$ for each $i$ and define an automorphism $g$ of $M$ by $\left.g\right|_{M_{i}}=g_{i}$. Since $g$ is invertible, so is $q(g)$ and since $p$ is onto, there exists $h \in \prod_{i=1}^{n}$ End $_{A} M_{i}$ such that $p(h)=q(g)^{-1}$. Clearly $f=g \cdot j(h)$ satisfies $f\left(M_{i}\right)=M_{i}^{\prime}$ and $\bar{f}=1$.

(iii) By (ii), if $M_{1} \cong M_{1}^{\prime}$, there exists $f \in$ Aut $_{A} M$ such that $f\left(M_{1}\right)=M_{1}^{\prime}$, $f\left(\oplus_{i=2}^{n} M_{i}\right)=\bigoplus_{i=2}^{n} M_{i}^{\prime}$ and $\bar{f}=1$. It follows easily that $e_{1}^{\prime}=f e_{1} f^{-1}$ and therefore $\bar{e}_{1}^{\prime}=\bar{e}_{1}$.

Conversely suppose $\bar{e}_{1}^{\prime}=\bar{e}_{1}$. By Krull-Schmidt theorem, $M_{1}^{\prime} \cong M_{i}$ for some $i$. By the first part of this proof, $\bar{e}_{1}^{\prime}=\bar{e}_{i}$. Hence $\bar{e}_{i}=\bar{e}_{1}$ and so $i=1$.

Proof of Theorem 2. (i) Write $V_{A_{1}}=\oplus_{i=1}^{r} W_{i}$ with the $W_{i}$ indecomposable. Since $V$ is a direct summand of $W^{A}, V_{A_{1}}$ is a summand of $\left(W^{A}\right)_{A_{1}} \cong \bigoplus_{s \in S} a_{s} \otimes W$. By Krull-Schmidt theorem, each $W_{i}$ is isomorphic to some $a_{s} \otimes W$. 
(ii) Changing notations write $V_{A_{1}}=\bigoplus_{i=1}^{n} m_{i} W_{i}$ where $m_{i} W_{i}$ denotes the direct sum of $m_{i}$ copies of $W_{i}$ and $W_{i} \neq W_{i}$ if $i \neq j$. By (i), $W_{i} \equiv a_{s} \otimes W$ for some $s$. Applying $a_{\text {s }}$ to $V$, we get:

$$
\bigoplus_{i=1}^{n} m_{i} W_{i} \cong V_{A_{1}}=\left(a_{s} V\right)_{A_{1}} \cong \bigoplus_{i=1}^{n} m_{i}\left(a_{s} \otimes W_{i}\right)
$$

Comparing the multiplicities of $W_{i}$ in both decompositions, we get $m_{i}=m_{1}$. The same argument applied with an arbitrary $a_{s}$ shows that $a_{s} \otimes W$ must be isomorphic to some $W_{i}$. Therefore, by definition of $T,\left\{a_{s_{i}} \otimes W \mid i=1, \ldots, n\right\}$ is a complete set of non-isomorphic conjugates of $W$.

(iii) Let $E=\operatorname{End}_{A_{1}} V$ and $\bar{E}=E / \mathrm{rad}(E)$. The group $S$ acts on $E$ via $s f=$ $a_{s} f a_{s}^{-1}$ and induces an action on $\bar{E}$ which necessarily permutes the primitive central idempotents of $\bar{E}$.

Let $V_{\mathrm{A}_{1}}=\bigoplus_{i=1}^{n} U_{i}$ be a decomposition of $V_{\mathrm{A}_{1}}$ into homogeneous components, corresponding to idempotents $e_{1}, \ldots, e_{n}$. Assume $W$ is a summand of $U_{1}$. For $s \in S, V_{A_{1}}=\bigoplus_{i=1}^{n} a_{s} U_{i}$ is also a decomposition of $V_{A_{1}}$ into homogeneous components, corresponding to idempotents $a_{s} e_{i} a_{s}^{-1}=s e_{i}$. By Proposition 3(i), $\left\{\bar{e}_{1}, \ldots, \bar{e}_{n}\right\}$ are the primitive central idempotents of $\bar{E}$. Since $a_{s} U_{1} \cong a_{s} \otimes U_{1} \cong U_{i}$ for some $i$, we have $s \bar{e}_{1}=\bar{e}_{i}$ by Proposition 3(iii). Moreover each $U_{i}$ is isomorphic to some $a_{s} U_{1}$ by part (i) and (ii). This implies that $S$ acts transitively on the set $\left\{\bar{e}_{1}, \ldots, \bar{e}_{n}\right\}$. Since $W$ is a summand of $U_{1}, T$ is the stabilizer of $\bar{e}_{1}$ (again by Proposition 3(iii)).

Now since $V$ is projective relative to $A_{1}$, there exists $v \in \operatorname{End}_{A_{1}} V$ such that $\operatorname{Tr}_{\mathrm{S}}(v)=1$. Let $u=\sum_{i=1}^{n} r_{i} v$ where $r_{1}, \ldots, r_{n}$ are representatives of the cosets $T r$. Then $\operatorname{Tr}_{T}(u)=\sum_{t \in T} t u=\operatorname{Tr}_{S}(v)=1$. Moreover $\bar{u}$ commutes with $\bar{e}_{i}$ for $\bar{e}_{i}$ is central. Therefore the hypotheses of Theorem 1 are satisfied. It follows that there exist orthogonal idempotents $f_{1}, \ldots, f_{n}$ of $E$ (lifting $\bar{e}_{1}, \ldots, \bar{e}_{n}$ ) which are permuted transitively by $S$ and such that $T$ stabilizes $f_{1}$.

By Proposition 3(i), the modules $f_{i} V_{\mathrm{A}_{1}}$ are homogeneous components of $V_{\mathrm{A}_{1}}$. The equation $f_{i}=s f_{1}=a_{\mathrm{s}} f_{1} a_{s}^{-1}$ means exactly that $a_{s}\left(f_{1} V_{\mathrm{A}_{1}}\right)=f_{i} V_{\mathrm{A}_{1}}$. This completes the proof of part (iii).

(iv) Since $\left\{a_{t} \mid t \in T\right\}$ stabilizes $U_{1}=f_{1} V_{A_{1}}, U_{1}$ is a $B$-module. Now $V=$ $\bigoplus_{i=1}^{n} a_{s_{i}} U_{1}$ which is the definition of an induced module. Finally $U_{1}$ is indecomposable otherwise $V$ would be decomposable.

Counter-example. Without the assumption of relative projectivity for $V$, Theorem 2 does not hold any more. Take $K$ a field of characteristic $2, G=$ $C_{4}, N=C_{2}$ and $V=K[X] /(X-1)^{3}$ (the generator of $C_{4}$ acting by multiplication by $X$ ). Then: $\operatorname{Res}_{N} V=S_{1} \oplus S_{2}$ where $S_{i}=K[Y] /(Y-1)^{i}$ (the generator of $C_{2}$ 
acting by multiplication by $Y$ ). Since $S_{1}$ and $S_{2}$ do not have the same dimension, they cannot be conjugate. In fact, the two primitive central idempotents of $\overline{\operatorname{End}_{K N} V}$ are fixed under the action of $S=G / N$, and each of them can be lifted in four ways in $\operatorname{End}_{K N} V$. But no idempotent of $\operatorname{End}_{K N} V$ is fixed by $S$.

Now we can recall the going-up theorem, which we shall prove to be equivalent to Theorem 2 .

THEOREM 4 (Conlon, Tucker, Ward $[2, \$ 19 \mathrm{C}]$ ). Let $A$ be an $S$-graded Clifford system over $R, W$ an indecomposable $A_{1}$-module, $T$ the inertial subgroup of $W$ and $B=\bigoplus_{t \in T} A_{t}$. If $W^{B}=\bigoplus_{i=1}^{m} Z_{i}$ is a decomposition of $W^{B}$ into indecomposable $B$-modules, then each $Z_{i}^{A}$ is an indecomposable $A$-module, that is $W^{A}=$ $\bigoplus_{i=1}^{m} Z_{i}^{\mathrm{A}}$ gives a decomposition of $\mathrm{W}^{\mathrm{A}}$ into indecomposable $\mathrm{A}$-modules.

Proof. The notation $X \mid Y$ will mean: $X$ is a direct summand of $Y$. Let $Z$ be an indecomposable summand of $W^{B}$. Since $T$ is the inertial subgroup of $W$, $\left(W^{B}\right)_{A_{1}}=|T| \cdot W$ and so $Z_{A_{1}}$ is a multiple of $W$. Since $Z \mid\left(Z^{A}\right)_{B}$, there exists an indecomposable summand $V$ of $Z^{A}$ such that $Z \mid V_{B}$. Then $V \mid W^{A}$ and $W \mid V_{A_{1}}$. By Theorem 2, there exists an indecomposable $B$-module $U$ such that $V \cong U^{A}$ and $U_{A_{1}}$ is a multiple of $W$. Now $U \mid\left(Z^{A}\right)_{B}$ because $V \mid Z^{A}$ and $U \mid\left(U^{A}\right)_{B}=V_{B}$. But $Z$ is the only indecomposable summand of $\left(Z^{A}\right)_{B}$ whose restriction to $A_{1}$ is a multiple of $W$, for $\left(Z^{A}\right)_{A_{1}}=\bigoplus_{i=1}^{n} a_{s_{1}} \otimes Z_{A_{1}}$ (where $\left\{s_{1}, \ldots, s_{n}\right\}$ is a set of coset representatives of $T$ in $S$ ) and $a_{s_{2}} \otimes Z_{A_{1}}$ is a proper conjugate of $Z_{A_{1}}$ (a multiple

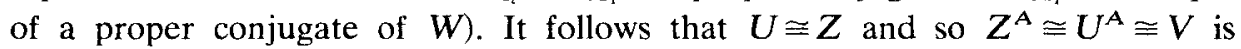
indecomposable.

Equivalence of Theorems 2 and 4 . If Theorem 4 is proved independently (e.g. by the proof of $[2, \S 19 \mathrm{C}])$, then Theorem 2 can be derived as corollary in the following way: Let $V$ be an indecomposable $A$-module which is a summand of $W^{A}$ for some indecomposable summand $W$ of $V_{A_{1}}$. Let $T$ be the inertial subgroup of $W$. By Theorem 4, there exists an indecomposable summand $U$ of $W^{B}$ such that $V=U^{\mathbf{A}}$. Now $U_{\mathbf{A}_{1}} \cong m W$ for some $m$ because $\left(W^{\mathbf{B}}\right)_{\mathbf{A}_{1}} \cong|T| W$. Then clearly $V \cong \bigoplus_{i=1}^{n} a_{s_{i}} \otimes U$ and $V_{A_{1}} \equiv \bigoplus_{i=1}^{n} m\left(a_{s_{i}} \otimes W\right)$ where $s_{1}, \ldots, s_{n}$ are coset representatives of $T$ in $S$. This completes the proof of Theorem 2 .

\section{Ground field extensions}

Let $K$ be a field and $A$ a finite dimensional $K$-algebra. Let $F$ be a finite Galois extension of $K$, with Galois group $\Pi$, and consider the $F$-algebra $F \otimes A$ (note that throughout this section $\otimes$ will always mean $\otimes_{K}$ ). Every element $\sigma \in \Pi$ induces a semi-linear automorphism $\sigma: F \otimes A \rightarrow F \otimes A$. If $W$ is an $F \otimes A$-module, one can define a new $F \otimes A$-module structure on $W$ by scalar extension via $\sigma$ (or 
equivalently restriction via $\sigma^{-1}$ ). Explicitly the new structure is given by $a \cdot w=$ $\sigma^{-1}(a) w, a \in F \otimes A, w \in W$. This module is called a Galois conjugate of $W$.

Now if $V$ is a finitely generated indecomposable $A$-module, then $F \otimes V$ has a natural structure of $F \otimes A$-module. Moreover, $\Pi$ acts on $F \otimes V$ via $\sigma(f \otimes v)=$ $\sigma f \otimes v, \sigma \in \Pi, f \in F, v \in V$. This action is semi-linear with respect to $F \otimes A$, i.e. $\dot{\sigma}(a w)=\sigma(a) \sigma(w), \sigma \in \Pi, a \in F \otimes A, w \in F \otimes V$. If $F \otimes V=\bigoplus_{i=1}^{n} W_{i}$ is a decomposition of $F \otimes V$ into homogeneous components, then so is $F \otimes V=\bigoplus_{i=1}^{n} \sigma W_{i}$. One can readily check that $\sigma W_{i}$ is a Galois conjugate of $W_{i}$. By Krull-Schmidt theorem, $\boldsymbol{\sigma} W_{i} \cong W_{j}$ for some $j$. Moreover, it is easy to see that for given $i$ and $j$, there exists $\sigma \in \Pi$ such that $\sigma W_{i} \cong W_{j}$. The purpose of this section is to derive from Theorem 1 a stronger result, namely that for a suitable choice of the submodules $W_{i}$, one can replace this isomorphism by an equality:

PROPOSITION 5. In the above notations, there exists a decomposition $F \otimes V=\bigoplus_{i=1}^{n} W_{i}$ of $F \otimes V$ into homogeneous components such that the modules $W_{i}$ are permuted transitively under the natural action of $I I$ on $F \otimes V$.

Proof. Let $E=\operatorname{End}_{\mathrm{A}} V$ and $\bar{E}=E / \operatorname{Rad} E$. Since $V$ is indecomposable, $\bar{E}$ is a division algebra containing $K$ in its center. Now $F \otimes E=E_{F d \otimes A}(F \otimes V)$ and let $\overline{F \otimes E}=F \otimes E / R \operatorname{ad}(F \otimes E)$. Since $F / K$ is separable, $\overline{F \otimes E} \cong F \otimes \bar{E}$. Let $F \otimes V=$ $\bigoplus_{i=1}^{n} W_{i}$ be a decomposition of $F \otimes V$ into homogeneous components corresponding to idempotents $e_{1}, \ldots, e_{n} \in F \otimes E$. The decomposition $F \otimes V=$ $\bigoplus_{i=1}^{n} \sigma W_{i}$ corresponds to the idempotents $\sigma e_{1} \sigma^{-1}, \ldots, \sigma e_{n} \sigma^{-1}$ (where $\sigma$ is viewed as a semi-linear automorphism of $F \otimes V$ ).

Now $\Pi$ acts on $F \otimes E$ via $\sigma \cdot(f \otimes e)=\sigma f \otimes e, \sigma \in \Pi, f \in F, e \in E$. We claim that $\sigma z \sigma^{-1}=\sigma \cdot z$ for all $z \in F \otimes E$. Indeed, if $z=f \otimes e, f \in F, e \in E$, and if $g \otimes v \in F \otimes V$, then:

$$
\begin{aligned}
\left(\sigma z \sigma^{-1}\right)(g \otimes v) & =\sigma(f \otimes e)\left(\sigma^{-1} g \otimes v\right)=\sigma\left(f \cdot \sigma^{-1} g\right) \otimes e v=\sigma f \cdot g \otimes e v \\
& =(\sigma f \otimes e)(g \otimes v)=(\sigma \cdot z)(g \otimes v)
\end{aligned}
$$

It follows that $\left\{\sigma \cdot e_{1}, \ldots, \sigma \cdot e_{n}\right\}$ are the idempotents corresponding to the decomposition $F \otimes V=\bigoplus_{i=1}^{n} \sigma W_{i}$. By Proposition 3(i), $\left\{\bar{e}_{1}, \ldots, \bar{e}_{n}\right\}=$ $\left\{\overline{\sigma \cdot e_{1}}, \ldots, \overline{\left.\sigma \cdot e_{n}\right\}}\right.$ is the set of primitive central idempotents of $F \otimes \bar{E}$. Now $\Pi$ acts transitively on $\left\{\bar{e}_{1}, \ldots, \bar{e}_{n}\right\}$ for if $\left\{\bar{e}_{1}, \ldots, \bar{e}_{k}\right\}$ is a $\Pi$-orbit, then $\bar{e}=\sum_{i=1}^{k} \bar{e}_{k}$ is an idempotent, invariant under $\Pi$, hence lies in $K \otimes \bar{E}=\bar{E}$. Since 1 is the only idempotent of $\bar{E}$, we get $\bar{e}=1$ and so $k=n$.

Since $F / K$ is separable, $T_{F / K}$ is surjective. Therefore there exists $x \in F$ such that $\operatorname{Tr}_{F / K}(x)=\sum_{\sigma \in \Pi} \sigma x=1$. In particular, if $\Omega$ denotes the stabilizer of $\bar{e}_{1}$ and $\sigma_{1}, \ldots, \sigma_{n}$ are coset representatives of $\Omega$ in $\Pi$, then $u=\sum_{i=1}^{n} \sigma_{i} x$ satisfies $\sum_{\omega \in \Omega} \omega u=1$. Also $u \otimes \overline{1} \in F \otimes \bar{E}$ commutes with every $\bar{e}_{i}$. Therefore $u \otimes 1 \in F \otimes E$ 
satisfies the hypotheses of Theorem 1 . Consequently $\left\{\bar{e}_{1}, \ldots, \bar{e}_{n}\right\}$ lifts to a set of orthogonal idempotents $f_{1}, \ldots, f_{n}$ of $F \otimes E$ which are permuted transitively by $\Pi$ and such that $\sum_{i=1}^{n} f_{i}=1$. By Proposition 3(i), the modules $W_{i}^{\prime}=f_{i}(F \otimes V)$ are homogeneous components of $F \otimes V$. Finally, since $\sigma f_{i}=f_{j}$ for some $j$, we have:

$$
\sigma W_{i}^{\prime}=\sigma\left(f_{i}(F \otimes V)\right)=\left(\sigma f_{i} \sigma^{-1}\right)(F \otimes V)=\left(\sigma \cdot f_{i}\right)(F \otimes V)=f_{i}(F \otimes V)=W_{i}^{\prime}
$$

Remarks. 1) If one replace homogeneous components of $F \otimes V$ by indecomposable summands, then one must consider sets of primitive idempotents $\left\{\bar{e}_{1}, \ldots, \bar{e}_{n}\right\}$ of $\vec{E}$ instead of primitive central idempotents of $\vec{E}$. If one can show that there exists such a set which is stable under the action of $\Pi$ (this happens quite often), then the whole proof works without change, so that there exists a decomposition $F \otimes V=\bigoplus_{i=1}^{n} W_{i}$ into indecomposable submodules such that the modules $W_{i}$ are permuted transitively under the natural action of $\Pi$ on $F \otimes V$.

2) Proposition 5 holds more generally if one replaces the field $K$ by a complete discrete valuation ring $R$ and the extension $F$ by an unramified Galois extension $S$ (so that the Galois group of $S / R$ is isomorphic to the Galois group of the residue field extension). Moreover, $A$ must be an $R$-algebra which is finitely generated as $R$-module.

3) The similarity between restriction to a normal subgroup (Theorem 2) and ground field Galois extension (Proposition 5) extends a little further. If $\boldsymbol{\Omega}$ denotes the stabilizer of the homogeneous component $W_{1}$ of $F \otimes V$ and if $L$ is the fixed field of $\Omega$, then $W_{1}$ is realizable over $L$, that is there exists an $L \otimes_{K} A$-module $U$ such that $F \otimes_{x} U=W_{1}$. Moreover, by analogy with part (iv) of Theorem 2 (replacing group induction by scalar restriction), one can easily show that $V \cong$ $\operatorname{Res}_{K} U$.

\section{REFERENCES}

[1] ClifFord, A. H., Representations induced in an invariant subgroup, Ann. Math. 38 (1937), 533-550.

[2] Curtis, C. W. and ReINER, I., Methods of Representation Theory, Vol. I, Wiley-Interscience, New York, 1981.

[3] THÉvenaz, J., Extensions of group representations from a normal subgroup, Comm. in Algebra, to appear.

Institut de Mathématiques

Université de Lausanne

CH-1015 Lausanne-Dorigny

Switzerland

Received May 3, 1982 